\title{
Ultrafast demagnetizing fields from first principles
}

\author{
Jacopo Simoni, ${ }^{*}$ Maria Stamenova, and Stefano Sanvito \\ School of Physics, AMBER and CRANN Institute, Trinity College, Dublin 2, Ireland \\ (Received 25 April 2016; revised manuscript received 17 November 2016; published 12 January 2017)
}

\begin{abstract}
We examine the ultrafast demagnetization process of iron-based materials, namely, $\mathrm{Fe}_{6}$ clusters and bulk bcc $\mathrm{Fe}$, with time-dependent spin-density functional theory (TDSDFT). The magnetization continuity equation is reformulated and the torque due to the spin-current divergence is written in terms of an effective time-dependent kinetic magnetic field, an object already introduced in the literature. Its time evolution, as extracted from the TDSDFT simulations, is identified as one of the main sources of the local out-of-equilibrium spin dynamics and it plays a major role in the demagnetization process in combination with the spin orbit interaction. Such demagnetization is particularly strong in hot spots where the kinetic torque is maximized. Finally, we find the rate of demagnetization in $\mathrm{Fe}_{6}$ to be strongly dependent on the direction of polarization of the exciting electric field and this can be linked to the out-of-equilibrium distribution of the kinetic field in two comparative cases.
\end{abstract}

DOI: 10.1103/PhysRevB.95.024412

\section{INTRODUCTION}

The search for practical solutions for increasing the speed of manipulation of magnetic bits is essential for the progress of modern information and communication technology. It has been shown that there is an upper limit to the speed of the magnetization switching process when this is driven by a magnetic field $[1,2]$. An increase in power absorption beyond this limit and for higher magnetic-field amplitudes push a spin system out of equilibrium into a chaotic behavior and the switching speed decreases. For this reason the discovery made by Beaurepaire et al. [3] that a ferromagnetic Ni film could be demagnetized by a 60 -fs optical laser pulse has attracted a great deal of interest and was the seed of a new field, now called femtomagnetism.

In a standard pump-probe experiment the system is initially excited by an optical pulse (pump) and then the magnetization dynamics is monitored by analyzing a second signal (probe) [4,5]. Depending on the minimal delay between the pump and the probe, one can analyze the demagnetization process at different time scales and thus observe the dissipation mechanisms active at that particular time. The interpretation of the results is, however, a complicated matter. In general, for demagnetization processes observed on a time scale ranging from nanoseconds to $100 \mathrm{ps,}$ one considers an empirical three-temperature model [6], where electrons, spins, and phonons define three energy baths, all interacting with each other. In contrast, ultrafast spin dynamics, taking place within a few hundred femtoseconds, is yet not described in terms of a single unified scheme and various models for the demagnetization process have been advanced. These include fully relativistic direct transfer of angular momentum from the light to the spins [7,8], dynamical exchange splitting [9], electron-magnon spin-flip scattering [10], electron-electron spin-flip scattering [11], and laser-generated superdiffusive spin currents [12].

Given the complexity of the problem, ab initio methods, resolved in the time domain, provide a valuable tool to probe the microscopic aspects of the ultrafast spin dynamics of real

*simonij@tcd.ie magnetic materials by means of time-dependent simulations. In this work we apply time-dependent spin-density functional theory (TDSDFT) $[13,14]$ in its semirelativistic, noncollinear, spin-polarized version to analyze the ultrafast laser-induced demagnetization of two ferromagnetic transition metal systems: a $\mathrm{Fe}_{6}$ cluster (see Fig. 1) and bulk bcc Fe. Recently, within a similar theoretical description, it has been demonstrated that the spin-orbit ( $\mathrm{SO}$ ) interaction plays a central role in the demagnetization process [15-17]. Furthermore, it was showed by us [18] that the laser-induced spin dynamics can be understood as the result of the interplay between the SO-coupling potential and an effective magnetic field. The so-called kinetic magnetic field $[19,20] \mathbf{B}_{\mathrm{kin}}(\mathbf{r}, t)$ originates from the presence of nonuniform spin currents in the system. In this work we focus on the anatomy of $\mathbf{B}_{\text {kin }}(\mathbf{r}, t)$ and we analyze in detail its role in the highly nonequilibrium process of ultrafast demagnetization.

The first formulation of the spin dynamics problem in transition metal systems was given in Refs. [19,20] by Katsnelson and Antropov, who laid down the foundation of density functional theory (DFT) -based spin dynamics, by deriving a set of equations of motion for the local magnetization vector. In those seminal works the magnetization dynamics was analyzed at the level of the adiabatic local spin-density approximation (ALSDA), but actual applications to real outof-equilibrium systems were not described. Our purpose is to clarify and quantify, through TDSDFT simulations at the level of the noncollinear ALSDA, the role played by $\mathbf{B}_{\mathrm{kin}}(\mathbf{r}, t)$ in the laser-induced ultrafast spin dynamics of transition metal ferromagnets.

The paper is divided into four main sections. In Sec. II we define the various fields that couple to the spins by isolating in the continuity equation only the terms that play a major role in the dynamical process. In Sec. III we present the results of the calculations for $\mathrm{Fe}_{6}$ clusters and show that hot spots for demagnetization are associated with larger misalignment of the kinetic magnetic field and the local spin density. This becomes more clear through evaluation of material derivatives. A demonstration of the effect of the polarization of the electric field on the rate of demagnetization of $\mathrm{Fe}_{6}$ is discussed in Sec. IV. In Sec. V we show that previous observations for $\mathrm{Fe}_{6}$ are valid for bulk bcc Fe as well. We summarize in Sec. VI. 

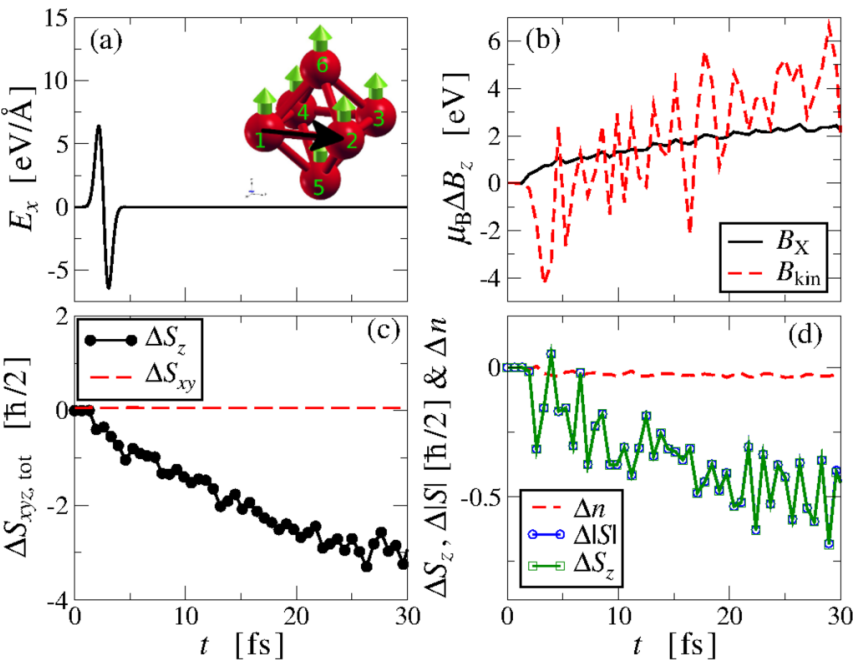

FIG. 1. (a) Typical electric-field pulse used to excite the $\mathrm{Fe}_{6}$ cluster with the black arrow indicating the direction of the field. The fluence of this pulse is $580 \mathrm{~mJ} / \mathrm{cm}^{2}$. (b) Time evolution of the $z$ component of $\mathbf{B}_{\text {kin }}$ and $\mathbf{B}_{\mathrm{X}}$ (exchange component of the field), with respect to their values at $t=0$ integrated over the system volume $\mu_{B} \mathbf{B}_{\mathrm{tot}}(t)=\mu_{B} \sum_{\mathrm{I}} \mathbf{B}_{\mathrm{I}}(t)$. (c) Time evolution of the variation of the total magnetization $\Delta S_{z}^{\text {tot }}(t)=\sum_{\mathrm{I}} \Delta S_{z}^{\mathrm{I}}(t)$ with respect to its initial value. (d) Time evolution on atomic site 6 of the magnetization variation along $z$ and of the electron density variation with respect to its value at $t=0$ integrated inside a sphere of radius $R=0.9 \AA$.

In the Appendix we present a detailed derivation of the spin continuity equation.

\section{THEORY}

We consider the TDSDFT problem within the ALSDA for a spin-polarized system excited by an electric-field pulse. If one neglects second-order contributions arising from the solution of the coupled Maxwell-Schrödinger system of equations, the dynamics will be governed by the usual set of time-dependent Kohn-Sham (KS) equations

$$
i \hbar \frac{d}{d t} \psi_{j}^{\mathrm{KS}}(\mathbf{r}, t)=H_{\mathrm{KS}}(\mathbf{r}, t) \psi_{j}^{\mathrm{KS}}(\mathbf{r}, t) .
$$

In Eq. (1) $\psi_{j}^{\mathrm{KS}}(\mathbf{r}, t)$ are the KS orbitals and the KS Hamiltonian $H_{\mathrm{KS}}(\mathbf{r}, t)$ can be expressed in the velocity gauge formulation and the minimal coupling substitution as

$$
\begin{aligned}
H_{\mathrm{KS}}(\mathbf{r}, t)= & \frac{1}{2 m}\left(-i \hbar \nabla-\frac{q}{c} \mathbf{A}_{\mathrm{ext}}(t)\right)^{2} \\
& -\mu_{\mathrm{B}} \hat{\boldsymbol{\sigma}} \cdot \mathbf{B}_{\mathrm{s}}[n, \mathbf{m}](\mathbf{r}, t)+v_{\mathrm{s}}[n](\mathbf{r}, t),
\end{aligned}
$$

where

$$
\begin{aligned}
v_{\mathrm{S}}[n](\mathbf{r}, t)= & \int d^{3} \mathbf{r}^{\prime} \frac{n\left(\mathbf{r}^{\prime}\right)}{\left|\mathbf{r}-\mathbf{r}^{\prime}\right|}+v_{\mathrm{XC}}^{\mathrm{ALSDA}}[n](\mathbf{r}, t) \\
& +\sum_{I} V_{\mathrm{PP}}^{I}\left(\left|\mathbf{r}-\mathbf{R}_{I}\right|\right)
\end{aligned}
$$

and

$$
\mathbf{B}_{\mathrm{s}}[n, \mathbf{m}](\mathbf{r}, t)=\mathbf{B}_{\mathrm{XC}}^{\mathrm{ALSDA}}[n, \mathbf{m}](\mathbf{r}, t)+\mathbf{B}_{\mathrm{ext}}(\mathbf{r}, t) .
$$

Here $v_{\mathrm{s}}(\mathbf{r}, t)$ represents the usual noninteracting $\mathrm{KS}$ potential and the full noninteracting magnetic field $\mathbf{B}_{\mathrm{s}}(\mathbf{r}, t)$ consists of the external one $\mathbf{B}_{\text {ext }}(\mathbf{r}, t)$ and the exchange-correlation (XC) magnetic field $\mathbf{B}_{\mathrm{XC}}^{\mathrm{ALSDA}}(\mathbf{r}, t)$. In the equations above $m$ is the electron mass, $q$ the electron charge, $c$ the speed of light, $\mathbf{A}_{\text {ext }}(t)$ the vector potential associated with the external magnetic field, $\hat{\sigma}$ the spin operator, $\mu_{\mathrm{B}}$ the Bohr magneton, $n$ the electron density, and $\mathbf{m}$ the magnetization density. Then $v_{\mathrm{s}}(\mathbf{r}, t)$ is decomposed into a Hartree contribution, an XC correlation one $v_{\mathrm{XC}}^{\mathrm{ALSDA}}[n](\mathbf{r}, t)$, and an ionic pseudopotential $V_{\mathrm{PP}}^{I}\left(\left|\mathbf{r}-\mathbf{R}_{I}\right|\right)$. For a fully relativistic, norm-conserving pseudopotential the SO coupling enters the KS equations in the form [21]

$$
\begin{aligned}
V_{\mathrm{PP}}^{I}\left(\left|\mathbf{r}-\mathbf{R}_{I}\right|\right)= & \sum_{l}\left(\bar{V}_{l}^{I}(\mathbf{r})+\frac{1}{4} V_{l}^{I, \mathrm{SO}}(\mathbf{r})\right. \\
& \left.+\sum_{m=-l}^{l} V_{l}^{I, \mathrm{SO}}(\mathbf{r}) \hat{\mathbf{L}}_{I} \cdot \hat{\mathbf{S}}|I, l, m\rangle\langle I, l, m|\right) .
\end{aligned}
$$

In Eq. (5) the orbital momentum operator associated with the $I$ th atomic center is $\hat{\mathbf{L}}_{I}$, while the vectors $\{|I, l, m\rangle\}$ are the associated set of spherical harmonics centered on that given atomic position. In Eq. (5) $V_{l}^{I, S O}(\mathbf{r})$ defines a generalized space-dependent $\mathrm{SO}$ coupling parameter providing a measure of the SO interaction strength close to the atomic site, while $\bar{V}_{l}^{I}(\mathbf{r})$ includes all the ionic relativistic corrections such as the Darwin and the mass correction terms. Within the ALSDA $v_{\mathrm{XC}}(\mathbf{r}, t)$ and $\mathbf{B}_{\mathrm{XC}}(\mathbf{r}, t)$ are local functions in time of the electron density and magnetization, which in turn are written in terms of the time-dependent KS orbitals

$$
\begin{gathered}
n(\mathbf{r}, t)=\sum_{j \in \mathcal{O}} \sum_{\sigma} \psi_{j \sigma}^{\mathrm{KS}}(\mathbf{r}, t)^{*} \psi_{j \sigma}^{\mathrm{KS}}(\mathbf{r}, t), \\
\mathbf{m}(\mathbf{r}, t)=\sum_{j \in \mathcal{O}} \sum_{\alpha, \beta} \psi_{j \alpha}^{\mathrm{KS}}(\mathbf{r}, t)^{*} \boldsymbol{\sigma}_{\alpha, \beta} \psi_{j \beta}^{\mathrm{KS}}(\mathbf{r}, t),
\end{gathered}
$$

where $\mathcal{O}$ denotes occupied states. Starting from the set of time-dependent $\mathrm{KS}$ equations in (1), it is possible to derive an equation of motion for the magnetization, or a spin-continuity equation, in terms of the noninteracting KS observables. This reads

$$
\begin{aligned}
\frac{d}{d t} \mathbf{m}(\mathbf{r}, t)= & -\nabla \cdot \mathbf{J}_{\mathrm{KS}}(\mathbf{r}, t)+\mu_{\mathrm{B}} \mathbf{m}(\mathbf{r}, t) \times \mathbf{B}_{\mathrm{S}}(\mathbf{r}, t) \\
& +\mathbf{T}_{\mathrm{SO}}(\mathbf{r}, t),
\end{aligned}
$$

where $\mathbf{J}_{\mathrm{KS}}(\mathbf{r}, t)$ represents the noninteracting KS spin-current rank-2 tensor

$$
\mathbf{J}_{\mathrm{KS}}(\mathbf{r}, t)=\frac{\hbar}{2 m i} \sum_{j \in \mathcal{O}}\left(\psi_{j}^{\mathrm{KS} \dagger} \hat{\boldsymbol{\sigma}} \nabla \psi_{j}^{\mathrm{KS}}-\text { H.c. }\right)
$$

and the SO torque contribution reads

$$
\begin{aligned}
\mathbf{T}_{\mathrm{SO}}(\mathbf{r}, t)= & \sum_{I} \sum_{l, m_{1}, m_{2}} \sum_{j, \alpha, \beta}^{\mathcal{O}} V_{l}^{\mathrm{SO}}\left(\left|\mathbf{r}-\mathbf{R}_{I}\right|\right) \\
& \times\left\langle\psi_{j \alpha}^{\mathrm{KS}} \mid l, m_{1}, I\right\rangle\left\langle l, m_{1}, I\left|\mathbf{L}_{I}\right| l, m_{2}, I\right\rangle \times \boldsymbol{\sigma}_{\alpha \beta} \\
& \times\left\langle l, m_{2}, I \mid \psi_{j \beta}^{\mathrm{KS}}\right\rangle .
\end{aligned}
$$


The KS magnetic field $\mathbf{B}_{\mathrm{s}}(\mathbf{r}, t)$ is taken as in Eq. (4), which in the absence of an external magnetic field reduces to $\mathbf{B}_{\mathrm{XC}}(\mathbf{r}, t)$. In DFT there is a set of zero-force theorems stating that the interaction between the particles cannot generate a net force [22]. In the case of the exchange-correlation magnetic field we have the exact condition $\int d^{3} r \mathbf{m}(\mathbf{r}, t) \times \mathbf{B}_{\mathrm{XC}}(\mathbf{r}, t)=$ 0 , which is satisfied by the ALSDA. Combining this equality with the assumption that the currents at the system boundary are negligible allows us to conclude that the only source of global spin loss is the SO coupling torque $\mathbf{T}_{\mathrm{SO}}$ and that the spin lost during the temporal evolution is transferred to the orbital momentum of the system, which in turn is partially damped into the lattice (we consider frozen ions). Hence we have the relation

$$
\frac{d}{d t} \int_{\Omega} d^{3} r \mathbf{m}(\mathbf{r}, t)=\int_{\Omega} d^{3} r \mathbf{T}_{\mathrm{SO}}(\mathbf{r}, t),
$$

where the integration extends over the entire volume $\Omega$.

Within the ALSDA, the exchange-correlation functional satisfies also a local variant of the zero-torque theorem [23], which is not a property of the exact DFT functional [24-26]. According to this condition, $\mathbf{m}(\mathbf{r}, t) \times \mathbf{B}_{\mathrm{XC}}(\mathbf{r}, t)=0$ and therefore the exchange-correlation magnetic field cannot contribute, even locally, to the magnetization dynamics. This leads us to conclude that the local magnetization dynamics is solely the result of the interplay between the spin-polarized currents and the SO torque (in reality $\mathbf{B}_{\mathrm{XC}}$ can still contribute indirectly to the spin dynamics through a dynamical modification of the gap between up- and down-spin-polarized bands, which in turn may give rise to an enhancement of the spin dissipation via the spin-orbit-coupling channel). In order to elucidate this view further we make use of the hydrodynamical formalism applied to spin systems, which has already been introduced in Refs. [27,28]. This approach needs to be slightly modified in view of the fact that we are considering an effective Kohn-Sham system and not a set of independent spin particles. In fact, as it was already pointed out in Refs. [19,20], Eq. (8) can be written in a different form (the details of the derivation are shown in the Appendix)

$$
\begin{aligned}
& \frac{D}{D t} \mathbf{m}(\mathbf{r}, t)+\sum_{j \in \mathcal{O}} \boldsymbol{\nabla} \cdot \mathbf{v}_{j}(\mathbf{r}, t) \mathbf{m}_{j}(\mathbf{r}, t)=-\nabla \cdot \mathcal{D}(\mathbf{r}, t) \\
& \quad+\mu_{\mathrm{B}} \mathbf{m}(\mathbf{r}, t) \times \mathbf{B}_{\mathrm{eff}}(\mathbf{r}, t)+\mathbf{T}_{\mathrm{SO}}(\mathbf{r}, t)
\end{aligned}
$$

where a couple of new terms appears. In the equation $\frac{D}{D t}=\frac{d}{d t}+\mathbf{v} \cdot \nabla$ is a material derivative, $\mathbf{v}_{j}(\mathbf{r}, t)$ represents a single Kohn-Sham state velocity field (see the Appendix), and $\mathbf{m}_{j}(\mathbf{r}, t)=\psi_{j}^{\mathrm{KS} \dagger} \hat{\boldsymbol{\sigma}} \psi_{j}^{\mathrm{KS}}$. On the right-hand side of Eq. (12), in addition to the spin-orbit coupling torque $\mathbf{T}_{\mathrm{SO}}(\mathbf{r}, t)$ we have a new term $-\nabla \cdot \mathcal{D}(\mathbf{r}, t)$ that describes the spin dissipation in the system due to the internal motion of the spin currents. It can be interpreted as an effective spin-current divergence object involving only transitions among different Kohn-Sham states (interband transitions) [see Eq. (A16)]. Finally, the effective field $\mathbf{B}_{\text {eff }}$ is given by the sum of two terms $\mathbf{B}_{\text {eff }}=\mathbf{B}_{\mathrm{XC}}+\mathbf{B}_{\text {kin }}$, with $\mathbf{B}_{\mathrm{XC}}$ the exchange-correlation field and $\mathbf{B}_{\text {kin }}$ defined as [see Eq. (A25)]

$$
\mathbf{B}_{\text {kin }}(\mathbf{r}, t)=\frac{1}{\overline{\mathcal{F}} e}\left[\frac{\nabla n \cdot \nabla \mathbf{s}}{n}+\nabla^{2} \mathbf{s}\right]
$$

with spin vector field $\mathbf{s}(\mathbf{r}, t)=\frac{\mathbf{m}(\mathbf{r}, t)}{n(\mathbf{r}, t)}$.

Such a $\mathbf{B}_{\mathrm{kin}}(\mathbf{r}, t)$ field has only an instrumental role in the equations of motion for the spin density; a very similar expression was already introduced in some previous work. In Ref. [19] it is expressed in the form $\partial_{k} \frac{1}{n}\left(\mathbf{m} \times \partial_{k} \mathbf{m}\right)$, while in Ref. [20] it appears as $\frac{\nabla n \nabla \mathbf{m}}{n}$. The interpretation of $\mathbf{B}_{\text {kin }}$ may look quite obscure at first, however, in Refs. $[29,30]$ it was identified as a possible source of spin-wave excitations in the form of a spin-spin interaction potential.

In order to clarify this point, let us consider the Heisenberg interaction between two spins centered on atoms placed at a distance $d=|\mathbf{d}|$. We can assume naively, but reasonably, that the spin-spin interaction between the two spin distributions, computed at an arbitrary point $\mathbf{r}$ in space, may be expressed in the form

$$
H_{\mathrm{eff}}(\mathbf{r}) \simeq \mathbf{s}(\mathbf{r}-\mathbf{d} / 2) \cdot \mathbf{s}(\mathbf{r}+\mathbf{d} / 2),
$$

where it is more convenient for us to employ a spin field $\mathbf{s}(\mathbf{r})$, which describes the spin distribution in space, instead of an atom localized spin vector. Hence, $H_{\text {eff }}$ defines an effective single-particle Hamiltonian. By averaging over the number of electrons in the entire space we obtain

$$
\mathbf{S}_{1} \cdot \mathbf{S}_{2} \simeq \int_{\Omega} d^{3} r n(\mathbf{r}) \mathbf{s}(\mathbf{r}-\mathbf{d} / 2) \cdot \mathbf{s}(\mathbf{r}+\mathbf{d} / 2) .
$$

Then, by expanding the spin density in a Taylor series up to second order over the distance $d$ and then neglecting the zerothorder contribution (we focus our attention on the nonlocal term appearing in the expansion), after some straightforward rearrangement we arrive at

$$
\mathbf{S}_{1} \cdot \mathbf{S}_{2} \simeq-\frac{d^{2}}{4} \int_{\Omega} d^{3} r n(\mathbf{r}) \nabla \mathbf{s}(\mathbf{r}) \cdot \nabla \mathbf{s}(\mathbf{r}),
$$

which in turn becomes

$$
\begin{aligned}
\mathbf{S}_{1} \cdot \mathbf{S}_{2} \simeq & \frac{d^{2}}{4} \int_{\Omega} d^{3} r[-\nabla \cdot[n(\mathbf{r}) \mathbf{s}(\mathbf{r}) \cdot \nabla \mathbf{s}(\mathbf{r})] \\
& \left.+\mathbf{m}(\mathbf{r}) \cdot\left(\frac{\nabla n(\mathbf{r}) \cdot \nabla \mathbf{s}(\mathbf{r})}{n(\mathbf{r})}+\nabla^{2} \mathbf{s}(\mathbf{r})\right)\right] .
\end{aligned}
$$

Finally, by considering a sufficiently large integration volume, the use of the divergence theorem allows us to neglect all the boundary terms with the consequent final expression

$\mathbf{S}_{1} \cdot \mathbf{S}_{2} \simeq \frac{d^{2}}{4} \int_{\Omega} d^{3} r \mathbf{m}(\mathbf{r}) \cdot\left[\frac{\nabla n(\mathbf{r}) \cdot \nabla \mathbf{s}(\mathbf{r})}{n(\mathbf{r})}+\nabla^{2} \mathbf{s}(\mathbf{r})\right]$,

which remarkably resembles the result in Eq. (13) for the kinetic magnetic field. We can therefore tentatively interpret $\mathbf{B}_{\text {kin }}(\mathbf{r}, t)$ as an effective mean-field internal magnetic field, which plays a role in coupling the spins at different locations in the system analogously to the Heisenberg spin-spin interaction. 


\section{ANALYZING SPIN DYNAMICS FROM TDSDFT SIMULATIONS IN THE $\mathrm{Fe}_{6}$ CLUSTER}

Here we present the results of TDSDFT calculations, performed with the OCTOPUS code [31], where we simulate the ultrafast demagnetization process in iron-based ferromagnetic systems. In all those, at time $t=0$ the system is in its ground state. Then we apply an intense electric-field pulse with a duration of less than $10 \mathrm{fs}$, which initiates the dynamics. The pseudopotentials for Fe used in the calculations are fully relativistic, norm conserving, and generated using a multireference-pseudopotential scheme [32] at the level implemented in APE [33,34], which takes directly into account the semicore states. For the XC functional we employ the ALSDA with parametrization from Perdew and Wang [35]. Our simulations then consist in evolving in time the KS wave functions, i.e., in solving numerically the set of equations (1). The results are then interpreted through the magnetization continuity equation (12).

In Fig. 1 the extracted magnetization dynamics of a $\mathrm{Fe}_{6}$ magnetic cluster is presented. We use the LSDA ground-state geometry of $\mathrm{Fe}_{6}$ as extracted from Refs. [36,37] for which we reproduce the reported therein spin state $S=20 \hbar / 2$. The nuclei are kept stationary during the dynamics. In Fig. 1(c) we observe that the total loss of the $z$ component of the total magnetization $S_{z}^{\text {tot }}(t)$ is exactly equal to the variation in value of its module $\left|\mathbf{S}^{\text {tot }}\right|$ since the global noncollinear contribution is negligible. This indicates that the spin is not exchanged globally between the different components of the magnetization vector, but, according to Eq. (11), it is at least partially transferred into the orbital momentum of the system. We note that, due to the electrostatic interactions with the nuclei and due to the interaction with the laser field, the rotational invariance of the electronic system is broken and the total angular momentum is not conserved.

In Fig. 1(b) we observe that the average kinetic magnetic field (over the entire simulation box, for $\overline{\mathcal{F}}=1$ ) is comparable in magnitude to the exchange component. At the same time, $\mathbf{B}_{\mathrm{kin}, z}^{\text {tot }}$ shows a much more oscillatory behavior compared to $\mathbf{B}_{\mathrm{X}, z}^{\text {tot }}$. In particular, while $\mathbf{B}_{\mathrm{X}, z}^{\text {tot }}$ evolves smoothly in time following the action of the optical excitation, $\mathbf{B}_{\mathrm{kin}, z}^{\text {tot }}$ presents an abrupt variation at the onset of the electrical pulse. This is due to the fact that the laser pulse directly excites currents, through the term $-\nabla \cdot \mathcal{D}(\mathbf{r}, t)$ in Eq. (12), which in turn produces a modification of the gradients of the charge/spin density, even on a global scale since they are not conserved. Thus we observe large variations of $B_{\mathrm{kin}, z}^{\text {tot }}$. In addition, $B_{\mathrm{X}, z}$ can also oscillate very strongly locally, following the temporal variation of the densities, but in the evaluation of $B_{X, z}^{\text {tot }}$ these oscillations are averaged out given that the densities are approximately conserved over the entire simulation box. During the action of the pulse we see a tendency of the two fields to compensate each other, an effect strongly resembling the Lenz law. After the pulse, $\mathbf{B}_{\text {kin }}$ continues to oscillate dramatically with its average value, only slowly increasing. In contrast, $\mathbf{B}_{\mathrm{x}, z}^{\text {tot }}$ decreases (in absolute value) due the net dissipation of spin angular momentum.

Moving from an analysis of global quantities to probing locally the spin dynamics, in Fig. 1(d) we compare the magnetization and the electron density around atomic site 6 at the tip of the cluster [see inset of Fig. 1(a) for the numbering labels of all the cluster atoms]. We define local magnetization and charge associated with the particular atomic site I as

$$
\mathbf{S}^{\mathrm{I}}(t)=\frac{1}{\left|\mathcal{S}_{\mathrm{R}}^{\mathrm{I}}\right|} \int_{\mathcal{S}_{\mathrm{R}}^{\mathrm{I}}} d^{3} r \mathbf{m}(\mathbf{r}, t), \quad Q^{\mathrm{I}}(t)=\frac{1}{\left|\mathcal{S}_{\mathrm{R}}^{\mathrm{I}}\right|} \int_{\mathcal{S}_{\mathrm{R}}^{\mathrm{I}}} d^{3} r n(\mathbf{r}, t),
$$

where the integration volume $\mathcal{S}_{\mathrm{R}}^{\mathrm{I}}$ is a sphere of radius $\mathrm{R}$ centered at site I. Our results show that the loss of $S_{z}^{6}$ is not taking place just during the action of the external pulse, but it is rather distributed over the entire time evolution. This suggests that the spin-sink mechanism is not directly related to the coupling of the system to the laser field, but is rather intrinsic to the electron dynamics following the pulse. Furthermore, close to the atomic site, the temporal variation of the charge $Q^{6}$ is much smaller in magnitude and smoother than that of $S_{z}^{6}$. In addition, for long times $Q^{6}$ settles close to an average value, while $S_{z}^{6}$ continues to decrease. Hence the long-term spin dynamics is not the result of a net charge displacement from the region close to the ions to the interstitial space. These observations are valid for all the atomic sites in the cluster.

If we now consider the continuity equation for the electron density (see the Appendix for further explanation)

$$
\frac{D}{D t} n(\mathbf{r}, t)=-n(\mathbf{r}, t) \nabla \cdot \mathbf{v}(\mathbf{r}, t),
$$

where $\frac{D}{D t} n(\mathbf{r}, t)$ is the material derivative of the electron density

$$
\frac{D}{D t} n(\mathbf{r}, t)=\left(\frac{d}{d t}+\mathbf{v} \cdot \nabla\right) n(\mathbf{r}, t)
$$

From Fig. 1(d) we observe that during the action of the pulse the density variation in the vicinity of the atoms appears to be very small compared to the magnetization variation. We can therefore safely assume that in this spatial region $\dot{n}(\mathbf{r}, t) \simeq 0$, with at the same time $n(\mathbf{r}, t) \neq 0$. From these considerations we deduce that $\mathbf{v}(\mathbf{r}, t) \simeq 0$ is a reasonably good approximation for the velocity field in the vicinity of the atoms (this does not imply that the velocity field is exactly zero, but only that its effect on the spin dynamics in this particular case is negligible). The same argument is valid also for the state resolved density $n_{j}(\mathbf{r}, t)$, given that $\dot{n}(\mathbf{r}, t)=\sum_{j \in \mathcal{O}} \dot{n}_{j}(\mathbf{r}, t)$, the contribution of the local time derivative of the Kohn-Sham state density can be neglected. By applying the latter in Eq. (12) we finally obtain a relation that can be considered approximately valid in this spatial region of the simulation box,

$$
\frac{d}{d t} \mathbf{m}(\mathbf{r}, t) \simeq-\nabla \cdot \mathcal{D}+\mu_{B} \mathbf{m} \times \mathbf{B}_{\mathrm{kin}}+\mathbf{T}_{\mathrm{SO}},
$$

where the contribution to the spin dynamics due to the velocity-field term has been neglected. Note that here we have also used the condition $\mathbf{m}(\mathbf{r}, t) \times \mathbf{B}_{\mathrm{XC}}(\mathbf{r}, t)=0$, which is consequential to the LSDA. In addition, the decay of $\mathbf{B}_{\mathrm{XC}}$ during the evolution, is not so significant as to produce a dynamical modification of the gap between up- and down-spin states.

In Fig. 2 we compare the behavior of the kinetic field and of the local magnetization at two atomic sites, respectively: 1 (one of the atoms in the base plane of the bipyramid) and 6 (an atom at one of the apexes). It can be seen from Fig. 2(a) that these two 

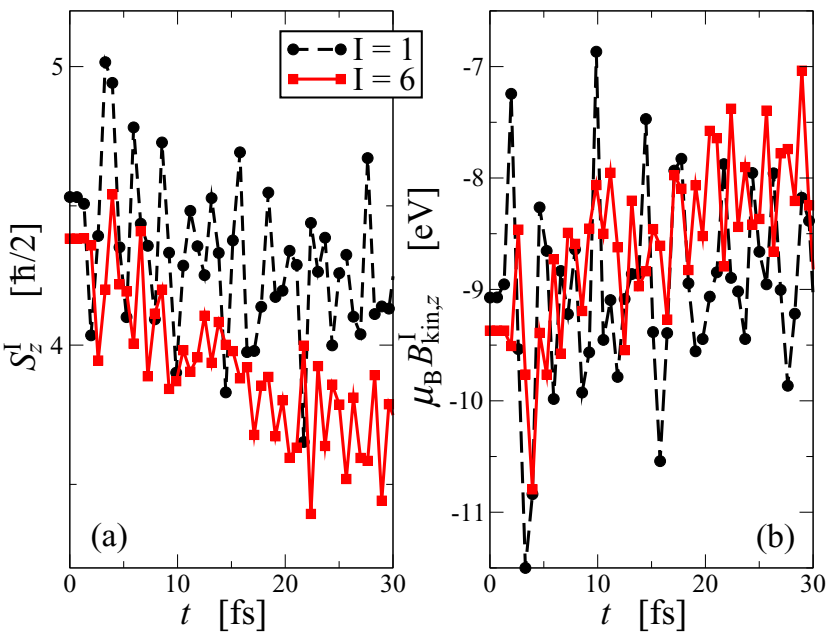

FIG. 2. Local spin dynamics of the $\mathrm{Fe}_{6}$ cluster: (a) time evolution of the magnetization $S_{z}^{\mathrm{I}}(t)$ around the atomic centers and (b) time evolution of the $z$ component of $\mathbf{B}_{\text {kin }}^{\mathrm{I}}(t)$. All the quantities are integrated inside a sphere of radius $R=0.9 \AA$ centered on the two atomic sites, where we have used $B_{z}^{\mathrm{I}}(t)=\frac{1}{\left|\mathcal{S}_{\mathrm{R}}^{\mathrm{I}}\right|} \int_{\mathcal{S}_{\mathrm{R}}^{\mathrm{I}}} d^{3} r B_{z}(\mathbf{r}, t)$.

sites present different rates of demagnetization. In particular, at site 6 the spin decay is considerably more prominent with respect to that observed at site 1 . In contrast, the fluctuations in $S_{z}^{\mathrm{I}}$ are significantly more pronounced for site 1 than for site 6. This can be understood from the fact that we have chosen here an electric pulse with polarization vector in the basal plane of the bipyramid. As such, the charge fluctuations for the atoms in the basal plane are expected to be much larger than those of the apical atoms. Finally, we note that $B_{\text {kin, } z}^{\mathrm{I}}(t)$ follows similar qualitative trends as $S_{z}^{\mathrm{I}}(t)$ [see Fig. 2(b)]. In fact, the average change following the excitation pulse is larger for site 6 (the one experiencing the larger demagnetization), but the fluctuations are more pronounced for site 1 [the one experiencing the larger fluctuations in $\left.S_{z}^{\mathrm{I}}(t)\right]$.

The correlation between the kinetic field and the magnetization loss is also rather evident in Fig. 3. There the time-averaged variations in the $x$ component of the two fields $\mathbf{m} \times \mathbf{B}_{\text {kin }}$ and $\dot{\mathbf{m}}(\mathbf{r}, t)$ are clearly comparable in magnitude and localized over the same regions of the simulation box. This demonstrates that the kinetic field can be considered as the main force driving the noncollinearity during the spin evolution. The fact that the contrast is stronger at the apex atoms (hot spots for demagnetization) agrees with Fig. 2(a), while the dipole-type patterns indicate how the longitudinal spin decays preserving global collinearity. The correlation between the $z$ components of $\mathbf{m} \times \mathbf{B}_{\text {kin }}$ and $\mathbf{m}(\mathbf{r}, t)$ is not as evident as that for the transverse component $x$. This is due to the fact that the $x$ and $y$ components of the field are much smaller compared to the $z$ one. Furthermore, the contribution to the spin dynamics along $z$ of the SO coupling, together with the internal dissipative term due to the spin currents, cannot be neglected.

In order to quantify the local noncollinearity we examine the evolution of the misalignment angle $\theta$ between the $z$ axis and the direction of the magnetic fields (averaged over spheres). It can be seen in Fig. 4 that at site 1 the averaged kinetic field and
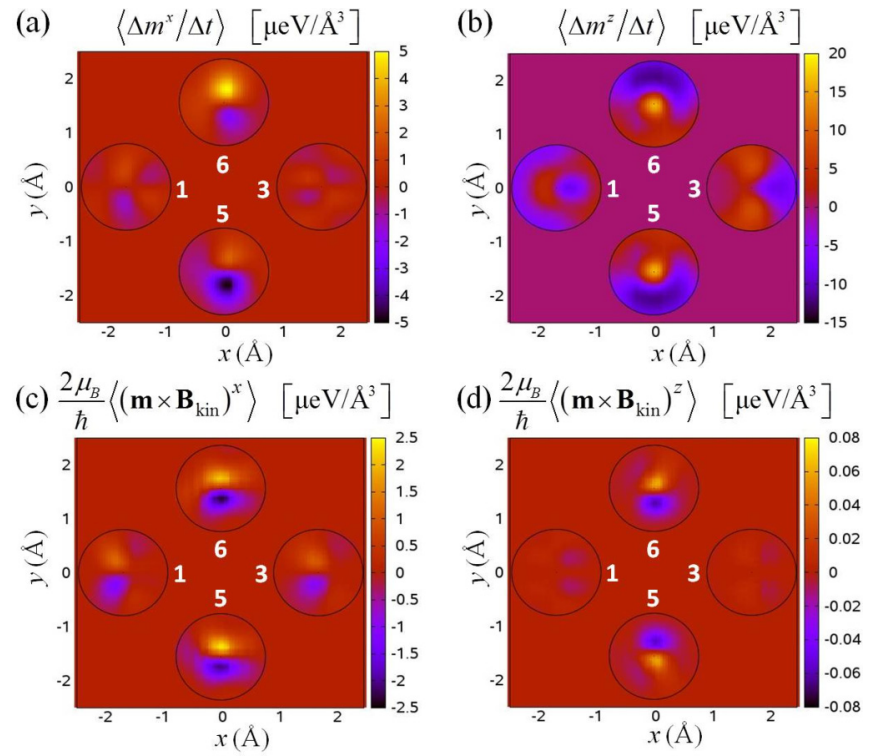

FIG. 3. Contour plots of the time- and space-averaged (in the direction perpendicular to the plane spanned by atoms $1,3,5$ and 6 , as indicated on the plot) observables evaluated only within spheres of radius $R=1.0 \AA$ around each atom: (a) and (b) the temporal variation of the spin density $\Delta \mathbf{m}^{(x, z)}(\mathbf{r}, t) / \Delta t$ for $\Delta t=0.1 \mathrm{fs}$ and (c) and (d) the $x$ and $z$ components of the second term on the right-hand side of Eq. (22).

the local spin deflect very little from the quantization axis and remain rather parallel to each other. The angle that $\mathbf{B}_{\text {kin }}^{1}(t)$ forms with the magnetization direction (the $\mathbf{m}^{1}$ direction) is practically negligible. Instead, at site $6, \mathbf{B}_{\text {kin }}^{6}(t)$ shows a significant deflection from the $z$ axis after the first 5 fs of the
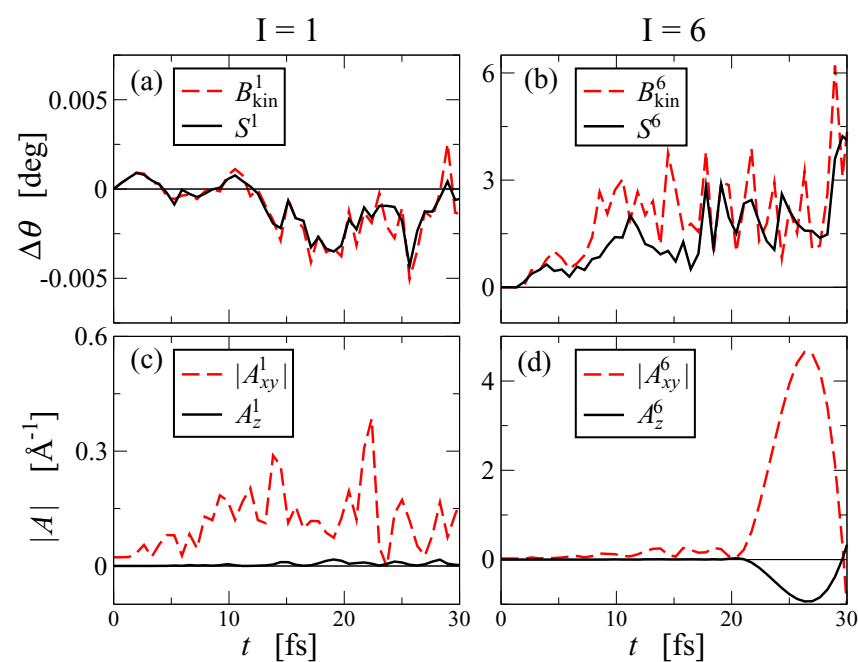

FIG. 4. Evolution of the spin noncollinearity in the $\mathrm{Fe}_{6}$ cluster. (a) Plot of $\Delta \theta=\theta(t)-\theta(0)$ at site 1 for the $\mathbf{B}_{\mathrm{XC}}[$ or $\mathbf{S}(t)]$ direction (black curve) and the $\mathbf{B}_{\text {kin }}$ direction (red dashed curve). (b) Same quantities as in (a) but calculated at atomic site 6. (c) Plot of $\sqrt{\overline{\mathcal{A}}_{1}^{2}+\overline{\mathcal{A}}_{2}^{2}}$, where $\overline{\mathcal{A}}=\sqrt{\sum_{i=1}^{3} \mathcal{A}_{i}^{2}}$ and $\mathcal{A}_{i}$ is introduced in Eq. (23), compared to $\overline{\mathcal{A}_{3}}$ at atomic site 1. (d) Same quantities as in (c) but calculated at atomic site 6 . The fields are measured within a sphere of radius $R=0.8 \AA$ centered on the atom center. 
evolution and so does the spin, without the two being parallel to each other. It is important to notice that the angle between magnetization $\mathbf{m}$ and $\mathbf{B}_{\text {kin }}$ starts to grow only after the action of the pulse. These results for atoms 1 and 6 are representative of all the other sites in the base plane or outside of it, respectively. The sites located in the plane, where $\mathbf{B}_{\text {kin }}^{\mathrm{I}}$ is mostly collinear, lose less magnetization with respect to the ones at the apices where instead the kinetic field shows a significant deflection from the magnetization axis and provides additional torque driving further demagnetization.

Analogous conclusions arise from the introduction of the concept of parallel transport, commonly used in differential geometry. This requires a proper definition of the covariant derivative obtained by comparing $\mathbf{s}(\mathbf{r}+d \mathbf{r})$ not with $\mathbf{s}(\mathbf{r})$, but with the value that the spin vector would have if it was translated from $\mathbf{r}$ to $\mathbf{r}+d \mathbf{r}$ while keeping the axes in the spin space fixed,

$$
D_{i} \mathbf{s}(\mathbf{r}, t)=d_{i} \mathbf{s}(\mathbf{r}, t)+\mathcal{A}_{i}(\mathbf{r}, t) \times \mathbf{s}(\mathbf{r}, t) .
$$

The connection field $\mathcal{A}(\mathbf{r}, t)$ provides a measure of the amount of noncollinearity accumulated in the translation of the spin vector from $\mathbf{r}$ to $\mathbf{r}+d \mathbf{r}$.

By using the previous expression to rewrite the first- and second-order spatial derivatives, the kinetic field of Eq. (13) can be divided into two components

$$
\mathbf{B}_{\mathrm{kin}}(\mathbf{r}, t)=\mathbf{B}_{\mathrm{kin}}^{0}(\mathbf{r}, t)+\delta \mathbf{B}_{\mathrm{kin}}(\mathbf{r}, t) .
$$

Here we have introduced

$$
\mathbf{B}_{\text {kin }}^{0}(\mathbf{r}, t)=\frac{1}{\overline{\mathcal{F}} e}\left[\frac{\nabla n(\mathbf{r}, t)}{n(\mathbf{r}, t)} \cdot D \mathbf{s}(\mathbf{r}, t)+D^{2} \mathbf{s}(\mathbf{r}, t)\right],
$$

which has no effect on the dynamics, having locally the same direction of the spin vector by construction, and

$$
\begin{aligned}
\delta \mathbf{B}_{\text {kin }}(\mathbf{r}, t)= & \frac{1}{\overline{\mathcal{F}} e} \sum_{i=1}^{3}\left[\frac{d_{i} n}{n}\left[-\mathbf{s}^{2} \mathcal{A}_{i}+\left(\mathbf{s} \cdot \mathcal{A}_{i}\right) \mathbf{s}\right]\right. \\
& -2\left[\mathbf{s}^{2} d_{i} \mathcal{A}_{i}-\mathbf{s}\left(\mathbf{s} \cdot d_{i} \mathcal{A}_{i}\right)\right]-2\left[\mathcal{A}_{i}\left(\mathbf{s} \cdot D_{i} \mathbf{s}\right)\right. \\
& \left.\left.-D_{i} \mathbf{s}\left(\mathbf{s} \cdot \mathcal{A}_{i}\right)\right]+4\left(\mathbf{s} \times \mathcal{A}_{i}\right)\left(\mathbf{s} \cdot \mathcal{A}_{i}\right)\right]
\end{aligned}
$$

According to Fig. 4, in the case of $\mathrm{Fe}_{6}$, the direction in the spin space of the connection tensor $\mathcal{A}_{i}$ for every $i$ component can be considered in a first approximation orthogonal to the direction of the spin vector $\mathbf{s}(\mathbf{r}, t)$, since its component along $z$ is considerably smaller than the components along $x$ and $y$. From this we could assume that $\mathbf{s} \cdot \mathcal{A}_{i} \simeq 0$ and we obtain the following simplified expression for $\delta \mathbf{B}_{\text {kin }}(\mathbf{r}, t)$ :

$$
\begin{aligned}
\delta \mathbf{B}_{\text {kin }}(\mathbf{r}, t)= & \frac{1}{\overline{\mathcal{F}} e} \sum_{i=1}^{3}\left[\left(-\frac{d_{i} n}{n} \mathbf{s}^{2}-2 \mathbf{s} \cdot D_{i} \mathbf{s}\right) \cdot \mathcal{A}_{i}\right. \\
& \left.-2 \mathbf{s}^{2} d_{i} \mathcal{A}_{i}\right] .
\end{aligned}
$$

This represents the part of $\mathbf{B}_{\text {kin }}$ that gives rise to a nonzero torque in Eq. (22).

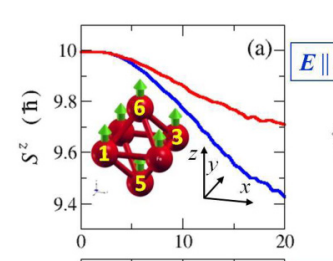

$\measuredangle\left(\vec{m}, \vec{B}_{k t n}\right) \quad(\mathrm{deg})$
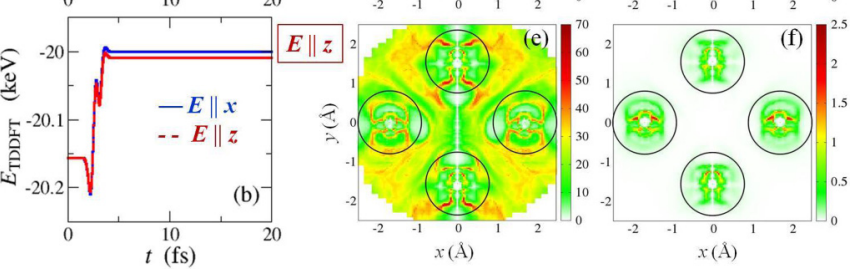

FIG. 5. Global (a) spin and (b) energy variation in $\mathrm{Fe}_{6}$ for two different excitations differing only by the direction of polarization of the electric-field pulse. Cartoon of the cluster with labels of the relevant atoms and a reference frame are depicted as insets. The contour plots represent (c) and (e) the distribution of the angle between $\mathbf{m}$ and $\mathbf{B}_{\text {kin }}$ in a plane through atoms $1,3,5$, and 6 (as in Fig. 3) and (d) and (f) the perpendicular component of $\mathbf{m}$ with respect to $\mathbf{B}_{\text {kin }}$ averaged over the time of the simulation, for the two different excitations (a), (c), and (d) $\mathbf{E} \| \mathbf{x}$ and (b), (e), and (f) $\mathbf{E} \| \mathbf{z}$.

\section{DIRECTIONALITY OF THE DEMAGNETIZATION IN THE $\mathrm{Fe}_{6}$ CLUSTER}

In the previous sections we have revisited the concept of kinetic field, its derivation within DFT, and its properties as a major source of torque for the spin dynamics within the ALSDA. We have provided supporting evidence for the latter from TDSDFT calculations of the ultrafast demagnetization of the $\mathrm{Fe}_{6}$ cluster under the effect of a sub-10 fs electric-field pulse. Despite the conceptual clarity of $\mathbf{B}_{\text {kin }}$ as an instrumental object, very little useful physical intuition can be drawn from its definition in Eq. (13). Clearly, it is an intrinsic dynamic field that depends on the spin texture and its response to the external stimuli. It also feeds back into the dynamics of this same spin density, which is obviously a nonlinear process. In this section we seek to extend the evidential base for the connection between the torque due to $\mathbf{B}_{\text {kin }}$ and the rate of demagnetization. Together with that we report a situation where the direction of the polarization vector of the electric field of the laser pulse alone has a significant effect on the demagnetization of a material (the $\mathrm{Fe}_{6}$ cluster).

Figure 5 shows a comparison between two simulations differing only by the direction (but notably not the magnitude) of the electric field applied. In one case this is in the $\mathbf{x}$ direction, which is oriented along the slightly longer side of the base of the bipyramid [36]; in the other simulation it is along $\mathbf{z}$, the direction connecting the two apex atoms 5 and 6 . The case $\mathbf{E} \| \mathbf{x}$ shows nearly 3 times faster demagnetization compared to the $\mathbf{E} \| \mathbf{z}$ one. Evidently, in the former situation more energy is absorbed by the cluster (an excess of about $8.6 \mathrm{eV}$ ); we show a comparison of the total energy shift due to the pulse in Fig. 5(b).

From Fig. 5(c) we observe that the amount of noncollinearity enclosed in a relatively small radius around the apex atoms does not significatively change in the two cases, suggesting that the intrasite noncollinear component of the spin vector is 
already present in the ground-state configuration. What really differs is the amount of intersite noncollinearity concentrated in the out-of-plane region. In the $\mathbf{E} \| \mathbf{x}$ case, if we examine the angle between $\mathbf{B}_{\text {kin }}(\mathbf{r}, t)$ and $\mathbf{m}(\mathbf{r}, t)$ averaged in time over the entire simulation along one particular cross-section plane (vertical through the base diagonal of the cluster as depicted in the inset), one can observe, in particular in the out-of-plane interstitial regions, a significantly larger amount of noncollinearity of the spin vector density, accumulating in the case of faster demagnetization.

Notably, there is a change in the symmetry between Figs. 5(c) and 5(e): In both cases no significant spin noncollinearity arises in the plane parallel to the field connecting the atomic centers. It is, however, important to notice that the amount of intrasite spin noncollinearity for the in-plane atoms is strongly dependent on the polarization direction of the applied laser field. In fact, in the case of $\mathbf{E} \| \mathbf{x}$ the temporally averaged angle between $\mathbf{s}$ and $\mathbf{B}_{\text {kin }}$ appears much higher with respect to the one computed in the $\mathbf{E} \| \mathbf{z}$ case. Figure 5(d) and 5(f) show that the non-collinear to $\mathbf{B}_{\text {kin }}$ spin density also quickly decays in the interatomic regions where the charge density is inherently lower.

Although $\mathbf{B}_{\text {kin }}$ is not the only torque generator and the SO contribution is significant too, the former plays a role in deflecting the spins in the system in a manner that correlates with the rate of global demagnetization. Importantly, our simulations clearly show that the demagnetization process is very anisotropic and particular directions of the exciting electric field may enhance the rate of demagnetization (this is only a demonstration of principle and the study of these effects is beyond the scope of this paper).

\section{DEMAGNETIZATION OF bcc Fe}

Finally, we present results of analogous simulations in bulk materials, namely, in bcc Fe, with the aim of demonstrating the qualitative universal role played by $\mathbf{B}_{\text {kin }}$ in the ultrafast demagnetization process. We consider bcc Fe in its ferromagnetic phase with total spin in the unit cell $S=4.85 \hbar / 2$ and with two atoms in it.

We employ a lattice parameter $a=2.9 \AA$, with a $4 \times 4 \times 4$ $k$-point grid. In Fig. 6(c) we show the demagnetization rate of the single unit cell after it has been excited with the electric-field pulse [Fig. 6(a)]. The green curve represents the demagnetization computed inside the unit cell and resembles in shape the sum of the magnetization variation $\sum_{\mathrm{I}} \Delta S_{z, \mathrm{I}}(t)$ calculated in the vicinity of the two Fe atoms, even if it is different in magnitude. This suggests that a large amount of spin is driven outside from the atomic integration region during the evolution. Similarly to the cluster, the dynamics of the on-site magnetization can be described in terms of a two-step process with an initial fast decay during the action of the external pulse, followed by a slower and noisy decrease in magnitude. The first fast decay may be attributed to the effect of the SO enhanced by the collapse of the effective field $\mathbf{B}_{\text {eff }}$ following the action of the laser pulse. In Fig. 6(b) the collapse after the first $5 \mathrm{fs}$ of the $z$ component of the effective field is quite clear, even if it appears to be more pronounced for the kinetic field $B_{\text {kin }, z}^{\mathrm{I}}(t)$ with respect to the exchange field $B_{\mathrm{X}, z}^{\mathrm{I}}(t)$. Similarly to the case of $\mathrm{Fe}_{6}$, the role played by $\nabla \cdot \mathcal{D}(\mathbf{r}, t)$ is
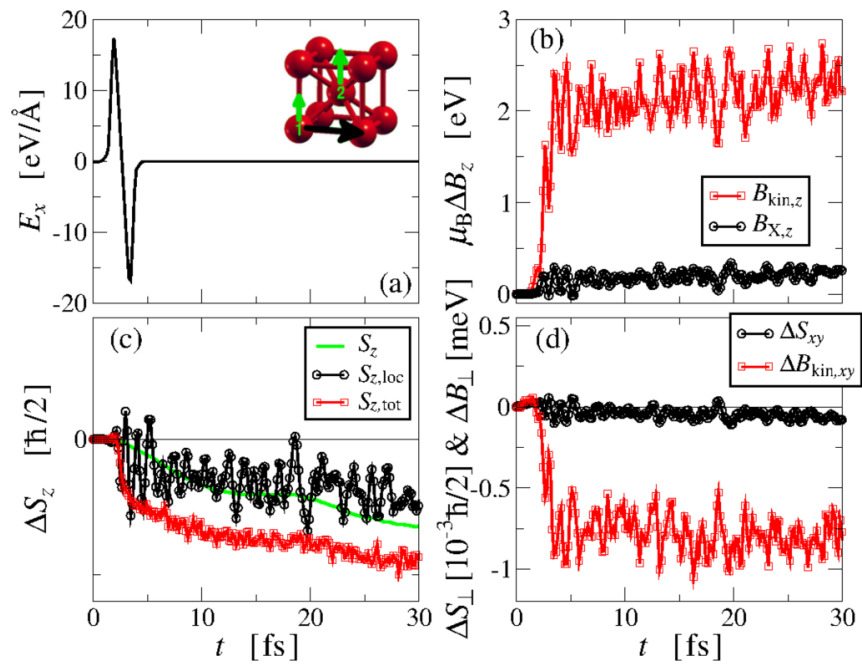

FIG. 6. Demagnetization of bcc Fe: (a) applied external electric field; (b) local value of $\Delta B_{\mathrm{kin}, z}^{\mathrm{I}}$ and $\Delta B_{\mathrm{x}, z}^{\mathrm{I}}$ around atom 1 ; (c) comparison between the value of the local magnetization $\Delta S_{z, \mathrm{I}}(t)$ around atom 1 (black curve), the total magnetization integrated around the two sites $\Delta S_{z, \mathrm{I}}(t)$ (red curve), and the total magnetization integrated inside the unit cell $\Delta S_{z}(t)$ (green curve); and (d) local value of $\sum_{\mathrm{I}} \Delta B_{\text {kin } x y}^{\mathrm{I}}(t)$ (red curve) and of $\Delta S_{x y}^{\mathrm{I}}(t)$, the noncollinear magnetization component for atom 1 . All the local quantities are calculated inside a sphere of radius $R=1 \AA$ centered on site 1 .

dominant during the action of the pulse, but after this initial phase the dynamics is dominated by intraband transitions and the interplay between the spin-orbit coupling and the effective field $\mathbf{B}_{\text {eff }}$ becomes dominant.

Figure 6(d) shows the evolution of the noncollinearity of the spin vector $\sum_{\mathrm{I}} S_{x y}^{\mathrm{I}}(t)$ and of the kinetic field $\sum_{\mathrm{I}} B_{\mathrm{kin}, x y}^{\mathrm{I}}(t)$. The level of correlation among the two quantities confirms the importance of the kinetic field in the evolution of the spin noncollinearity. The long tail of spin dissipation may be explained in terms of intraband spin-up/spin-down transitions through an Elliott-Yafet type of mechanism triggered by the scattering with the effective field $\mathbf{B}_{\text {eff }}$,

$$
A_{i \rightarrow f}=\left\langle\Psi_{n, \mathbf{k}_{1}}\left|\hat{\boldsymbol{\sigma}} \cdot \mathbf{B}_{\mathrm{eff}}\right| \Psi_{n, \mathbf{k}_{2}}\right\rangle .
$$

Here $A_{i \rightarrow f}$ represents the transition amplitude between two states with different $\mathbf{k}$ vector and in the presence of SO coupling with different mixing of up- and down-spin components.

\section{CONCLUSION}

In conclusion, we state the central result of our work, namely, that the equation of motion for the spin dynamics within the ALSDA of TDSDFT [see Eq. (8)] can be rewritten in the form of Eq. (12), by using a formalism borrowed from magnetohydrodynamics. We have analyzed the properties of the so-called kinetic magnetic field $\mathbf{B}_{\text {kin }}$ and its role in the ultrafast demagnetization process in two different systems: a ferromagnetic $\mathrm{Fe}_{6}$ cluster and bulk bcc Fe. The role of this field is particularly significant for processes far from equilibrium, such as the ultrafast demagnetization observed in transition metals.

In both systems studied the spin dynamics is the result of the interplay between the SO coupling and $\mathbf{B}_{\text {kin }}(\mathbf{r}, t)$, which, 
in general, is strongly coupled to the external pulse and highly nonuniform in space. We have shown that the spin loss locally correlates with $\mathbf{B}_{\mathrm{kin}}(\mathbf{r}, t)$. Through the concept of parallel transport and the definition of a connection tensor field $\mathcal{A}_{i}$, we have gained further insight into the evolution of the spin texture. As $\mathcal{A}_{i}$ describes the degree of spin rotation per infinitesimal spatial translation, it also provides a measure for the misalignment between the kinetic field and the spin texture. The regions with higher $\|\mathcal{A}\|$ correspond to stronger local demagnetization.

Finally, the effect of the direction of the polarization vector of the electric-field pulse has been studied for $\mathrm{Fe}_{6}$. We have found that clusters will demagnetize about twice as fast if the polarization vector is in the base plane and not vertical (through the apex atoms). Our analysis has shown a significant increase in the noncollinearity between $\mathbf{B}_{\text {kin }}(\mathbf{r}, t)$ and the spin density in the fast demagnetizing case. Such anisotropy, due to the electric dipole matrix elements for the valence electrons, is likely to occur in crystalline systems as well. During the application of the laser pulse, the rise of spin noncollinearity may be enhanced by the particular polarization direction of the laser pulse through the spin-orbit coupling and this effect combined with the collapse of the kinetic field may explain the initial spin loss. However, in both $\mathrm{Fe}_{6}$ and bcc Fe the magnetization loss is more prominent after the laser pulse has been reduced to zero. During this second phase of spin dissipation we anticipate microscopic differences between the spin decay observed in bcc Fe due to intraband transitions among states with different spin-up/spin-down mixture and the spin dynamics observed particularly at the apex atoms in $\mathrm{Fe}_{6}$ that is primarily driven by $\mathbf{B}_{\text {kin }}$ and directly related to the on-site intrinsic spin noncollinearity near the atomic sites.

\section{ACKNOWLEDGMENTS}

This work was funded by the European Commission project CRONOS (Grant No. 280879) and by Science Foundation Ireland (Grant No. 14/IA/2624). We gratefully acknowledge the DJEI/DES/SFI/HEA Irish Centre for High-End Computing for the provision of computational facilities. We also thank Professor E. K. U. Gross for valuable discussions.

\section{APPENDIX: DERIVATION OF THE HYDRODYNAMIC CONTINUITY EQUATION}

In this Appendix we show in some detail how Eq. (12) can be obtained by starting from the standard TDSDFT continuity equation (8). Here we follow the hydrodynamical formalism of quantum mechanics, where a single particle with spin is considered equivalent to a nonlinear vector field. In this type of hydrodynamics the quantum effects are separated as nonlinear terms and are described through effective quantum potentials (see Ref. [29]).

The formalism is based on the assumption that it is possible to describe the dynamical evolution of a single particle immersed in an external vector potential $\mathbf{A}(\mathbf{r}, t)$ through the so-called Madelung decomposition (see Ref. [38]) of the system wave function, in which the amplitude is translated into the probability density and the gradient of the phase determines the velocity field. A hydrodynamical description of the wave function was also obtained in Ref. [39] starting from the ordinary interpretation of quantum mechanics and by introducing an operator for the charge density and the current density.

The formalism was also later extended to the semirelativistic description (Pauli approximation) of a single particle in an external electromagnetic field in Ref. [27]. However, while in all the previous studies the main objective was to derive the single-particle dynamics of the spin- $1 / 2$ plasma, only recently has the study of the collective dynamical properties of the quantum plasma started to attract some interest (see Refs. [28,40]).

In deriving Eq. (12) for the spin density in the Kohn-Sham system we introduce also of the electron density $n(\mathbf{r}, t)$ and the velocity field $\mathbf{v}(\mathbf{r}, t)$. The equation of motion for the velocity field is not explicitly written here for the reasons explained in Sec. III.

The electron density is written in terms of the Kohn-Sham wave functions $\psi_{j}^{\mathrm{KS}}(\mathbf{r}, t)$ as

$$
n(\mathbf{r}, t)=\sum_{j \in \mathcal{O}} \psi_{j}^{\mathrm{KS}}(\mathbf{r}, t)^{\dagger} \psi_{j}^{\mathrm{KS}}(\mathbf{r}, t),
$$

while the spin density is

$$
\mathbf{s}(\mathbf{r}, t)=\frac{\sum_{j \in \mathcal{O}} \psi_{j}^{\mathrm{KS}}(\mathbf{r}, t)^{\dagger} \boldsymbol{\sigma} \psi_{j}^{\mathrm{KS}}(\mathbf{r}, t)}{n(\mathbf{r}, t)}
$$

and the covariant velocity field appears as

$$
\mathbf{v}(\mathbf{r}, t)=\frac{\hbar}{2 m i} \frac{\sum_{j \in \mathcal{O}}\left[\psi_{j}^{\mathrm{KS}}(\mathbf{r}, t)^{\dagger} \nabla \psi_{j}^{\mathrm{KS}}-\psi_{j}^{\mathrm{KS}} \nabla \psi_{j}^{\mathrm{KS}}(\mathbf{r}, t)^{\dagger}\right]}{n(\mathbf{r}, t)}-\frac{e}{m c} \mathbf{A}(\mathbf{r}, t) .
$$

By making use of the Kohn-Sham equations (1) the charge continuity equation can be written straightforwardly as

$$
\frac{d}{d t} n(\mathbf{r}, t)=-\frac{\hbar}{2 m i} \nabla \cdot \sum_{j \in \mathcal{O}}\left[\psi_{j}^{\mathrm{KS}}(\mathbf{r}, t)^{\dagger}(\vec{\nabla}-\overleftarrow{\nabla}) \psi_{j}^{\mathrm{KS}}(\mathbf{r}, t)\right]+\frac{e}{m c} \nabla \cdot[n \mathbf{A}(\mathbf{r}, t)]
$$

while for spin it is written in terms of $n(\mathbf{r}, t)$ and $\mathbf{s}(\mathbf{r}, t)$ as

$$
\frac{d}{d t}(n \mathbf{s})=-\frac{\hbar^{2}}{4 m i} \nabla \cdot \sum_{j \in \mathcal{O}}\left[\psi_{j}^{\mathrm{KS}}(\mathbf{r}, t)^{\dagger} \hat{\boldsymbol{\sigma}} \nabla \psi_{j}^{\mathrm{KS}}-\nabla \psi_{j}^{\mathrm{KS}}(\mathbf{r}, t)^{\dagger} \hat{\boldsymbol{\sigma}} \psi_{j}^{\mathrm{KS}}\right]+\frac{e}{m c} \sum_{j} \partial_{j}\left[A^{j} n \mathbf{s}\right]+\mu_{B} n\left(\mathbf{s} \times \mathbf{B}_{\mathrm{XC}}\right)+\mathbf{T}_{\mathrm{SO}}
$$


By evaluating explicitly the spatial partial derivative of the spin vector and multiplying it with the component $s_{i}$ we obtain the equality

$$
n s_{i} \partial_{l} s_{k}=s_{i} \sum_{j \in \mathcal{O}}\left[\partial_{l} \psi_{j}^{\mathrm{KS}}(\mathbf{r}, t)^{\dagger} \hat{\sigma}_{k} \psi_{j}^{\mathrm{KS}}+\psi_{j}^{\mathrm{KS}}(\mathbf{r}, t)^{\dagger} \hat{\sigma}_{k} \partial_{l} \psi_{j}^{\mathrm{KS}}\right]-\partial_{l} n s_{i} \cdot s_{k} .
$$

We now need to focus our attention on the first term on the right-hand side of Eq. (A6), by using the notation

$$
\mathcal{F}_{i k}(\mathbf{r}, t)=s_{i} \sum_{j \in \mathcal{O}}\left[\nabla \psi_{j}^{\mathrm{KS}}(\mathbf{r}, t)^{\dagger} \hat{\sigma}^{k} \psi_{j}^{\mathrm{KS}}+\psi_{j}^{\mathrm{KS}}(\mathbf{r}, t)^{\dagger} \hat{\sigma}^{k} \nabla \psi_{j}^{\mathrm{KS}}\right],
$$

which leads to

$$
\mathcal{F}_{i k}(\mathbf{r}, t)=\frac{1}{n} \sum_{j, r \in \mathcal{O}}\left[\psi_{r}^{\mathrm{KS} \dagger} \hat{\sigma}^{i} \psi_{r}^{\mathrm{KS}} \nabla \psi_{j}^{\mathrm{KS} \dagger} \hat{\sigma}^{k} \psi_{j}^{\mathrm{KS}}+\psi_{r}^{\mathrm{KS} \dagger} \hat{\sigma}^{i} \psi_{r}^{\mathrm{KS}} \psi_{j}^{\mathrm{KS} \dagger} \hat{\sigma}^{k} \nabla \psi_{j}^{\mathrm{KS}}\right] .
$$

The antisymmetric part $\mathcal{K}_{i k}(\mathbf{r}, t)$ of the tensor $\mathcal{F}_{i k}(\mathbf{r}, t)$, defined as $\mathcal{K}_{i k}=\mathcal{F}_{i k}-(i \leftrightarrow k)$, may be written as

$$
\mathcal{K}_{i k}(\mathbf{r}, t)=\frac{1}{n} \sum_{j, r \in \mathcal{O}} \sum_{\alpha, \beta, \alpha^{\prime}, \beta^{\prime}}\left[\psi_{r, \alpha}^{\mathrm{KS} *} \psi_{r, \beta}^{\mathrm{KS}} \sigma_{\alpha, \beta}^{[i,} \sigma_{\alpha^{\prime}, \beta^{\prime}}^{k]} \nabla \psi_{j, \alpha^{\prime}}^{\mathrm{KS} *} \psi_{j, \beta^{\prime}}^{\mathrm{KS}}+\psi_{r, \alpha}^{\mathrm{KS} *} \psi_{r, \beta}^{\mathrm{KS}} \sigma_{\alpha, \beta}^{\left[i, \sigma_{\alpha^{\prime}, \beta^{\prime}}^{k}\right.} \psi_{j, \alpha^{\prime}}^{\mathrm{KS} *} \nabla \psi_{j, \beta^{\prime}}^{\mathrm{KS}}\right] .
$$

By making use of the relation between Pauli matrices (see Ref. [29])

$$
\sigma_{\alpha, \beta}^{[i,} \sigma_{\alpha^{\prime}, \beta^{\prime}}^{k]}=i \sum_{s} \epsilon_{i k s}\left[\sigma_{\alpha, \beta^{\prime}}^{s} \delta_{\alpha^{\prime}, \beta}-\delta_{\alpha, \beta^{\prime}} \sigma_{\alpha^{\prime}, \beta}^{s}\right],
$$

we obtain the final expression for $\mathcal{K}_{i k}$ that can be split in two parts,

$$
\mathcal{K}_{i k}(\mathbf{r}, t)=\sum_{j, r \in \mathcal{O}} \mathcal{K}_{i k}^{(j, r)}(\mathbf{r}, t) \delta_{j, r}+\sum_{j \in \mathcal{O}} \sum_{r \neq j \in \mathcal{O}} \mathcal{K}_{i k}^{(j, r)}(\mathbf{r}, t),
$$

where we have introduced the tensor

$$
\mathcal{K}_{i k}^{(j, r)}(\mathbf{r}, t)=\frac{i}{n} \sum_{s} \epsilon_{i k s}\left[\psi_{r}^{\mathrm{KS} \dagger} \psi_{j}^{\mathrm{KS}}\left(\psi_{j}^{\mathrm{KS} \dagger} \hat{\sigma}^{s} \nabla \psi_{r}^{\mathrm{KS}}-\nabla \psi_{j}^{\mathrm{KS} \dagger} \hat{\sigma}^{s} \psi_{r}^{\mathrm{KS}}\right)+\psi_{r}^{\mathrm{KS} \dagger} \hat{\sigma}^{s} \psi_{j}^{\mathrm{KS}}\left(\nabla \psi_{j}^{\mathrm{KS} \dagger} \psi_{r}^{\mathrm{KS}}-\psi_{j}^{\mathrm{KS} \dagger} \nabla \psi_{r}^{\mathrm{KS}}\right)\right] .
$$

The procedure that we have followed up to now is formally exact. Then, in order to simplify the previous expression we substitute the Kohn-Sham ratio $\mathcal{F}_{j}=\frac{\psi_{j}^{\mathrm{KS} \dagger} \psi_{j}^{\mathrm{KS}}}{n(\mathbf{r}, t)}$ with its average over the various occupied states $\mathcal{F}_{j} \simeq \overline{\mathcal{F}}=\frac{\left\langle\psi_{j}^{\mathrm{KS} \dagger} \psi_{j}^{\mathrm{KS}}\right\rangle_{j}}{n(\mathbf{r}, t)}$. From the fact that, to a good degree of approximation, $\overline{\mathcal{F}} \simeq \frac{1}{N}$ with $N$ total number of particles in the system, we will consider from now on $\overline{\mathcal{F}}$ to be spatially homogeneous and constant in time. Then Eq. (A11) becomes

$$
\begin{aligned}
\mathcal{K}_{i k}(\mathbf{r}, t)= & i \overline{\mathcal{F}} \sum_{s} \epsilon_{i k s} \sum_{j \in \mathcal{O}}\left[\psi_{j}^{\mathrm{KS} \dagger} \hat{\sigma}^{s} \nabla \psi_{j}^{\mathrm{KS}}-\nabla \psi_{j}^{\mathrm{KS} \dagger} \hat{\sigma}^{s} \psi_{j}^{\mathrm{KS}}\right]+\frac{2 m \overline{\mathcal{F}}}{\hbar} \sum_{s} \epsilon_{i k s} \sum_{j \in \mathcal{O}} \psi_{j}^{\mathrm{KS} \dagger} \hat{\sigma}^{s} \psi_{j}^{\mathrm{KS}}\left[\mathbf{v}_{j}(\mathbf{r}, t)+\frac{e}{m c} \mathbf{A}(\mathbf{r}, t)\right] \\
& +\sum_{j \in \mathcal{O}} \sum_{r \neq j \in \mathcal{O}} \mathcal{K}_{i k}^{(j, r)}(\mathbf{r}, t) .
\end{aligned}
$$

In order to simplify the formalism we employ the notation $\mathcal{K}_{i k ; l}(\mathbf{r}, t)=n\left(s_{i} \partial_{l} s_{k}-s_{k} \partial_{l} s_{i}\right)$. Immediately from Eq. (A13) it follows that

$$
\frac{\hbar}{2 \overline{\mathcal{F}} m} n\left(s_{i} \partial_{l} s_{k}-s_{k} \partial_{l} s_{i}\right)=-\sum_{s} \epsilon_{i k s} \mathbf{J}_{\mathrm{KS}}^{s l}(\mathbf{r}, t)+\sum_{s} \epsilon_{i k s} \sum_{j \in \mathcal{O}} m_{j}^{s}(\mathbf{r}, t)\left[v_{j}^{l}(\mathbf{r}, t)+\frac{e}{m c} A^{l}(\mathbf{r}, t)\right]+\frac{\hbar}{2 \overline{\mathcal{F}}_{m}} \sum_{\substack{j \in \mathcal{O} \\ r \neq j \in \mathcal{O}}} \mathcal{K}_{i k ; l}^{(j, r)}(\mathbf{r}, t),
$$

where $\mathbf{m}_{j}$ and $\mathbf{v}_{j}$ define, respectively, the single Kohn-Sham state magnetization and velocity field. By employing the properties of the Levi-Cività tensor we have

$$
\frac{\hbar}{2 \overline{\mathcal{F}} m}\left(n \mathbf{s} \times \partial_{l} \mathbf{s}\right)^{n}=-\mathbf{J}_{\mathrm{KS}}^{n l}(\mathbf{r}, t)+\sum_{j \in \mathcal{O}} m_{j}^{n}(\mathbf{r}, t)\left[v_{j}^{l}(\mathbf{r}, t)+\frac{e}{m c} A^{l}(\mathbf{r}, t)\right]+\mathcal{D}_{n l}(\mathbf{r}, t),
$$

where we have introduced the new tensor quantity

$$
\mathcal{D}(\mathbf{r}, t)=-\sum_{j \in \mathcal{O}} \sum_{r \neq j \in \mathcal{O}}\left[\mathcal{F}_{r j} \mathcal{J}^{(j, r)}(\mathbf{r}, t)-\mathcal{F}_{j r} \mathbf{m}^{(r, j)}(\mathbf{r}, t) \otimes\left(\mathbf{v}^{(j, r)}(\mathbf{r}, t)+\frac{e}{m c} \mathbf{A}(\mathbf{r}, t)\right)\right] .
$$


Here $\mathcal{F}_{r j}=\frac{\psi_{r}^{\mathrm{KS} \dagger} \psi_{j}^{\mathrm{KS}}}{n(\mathbf{r}, t)}$ and the other many-particle objects are defined as

$$
\begin{gathered}
\mathcal{J}^{(j, r)}(\mathbf{r}, t)=-\frac{i \hbar}{2 m}\left[\psi_{j}^{\mathrm{KS} \dagger} \hat{\boldsymbol{\sigma}} \nabla \psi_{r}^{\mathrm{KS}}-\nabla \psi_{j}^{\mathrm{KS} \dagger} \hat{\boldsymbol{\sigma}} \psi_{r}^{\mathrm{KS}}\right], \\
\mathbf{v}^{(j, r)}(\mathbf{r}, t)=\frac{\hbar}{2 m i} \frac{\psi_{j}^{\mathrm{KS} \dagger} \nabla \psi_{r}^{\mathrm{KS}}-\nabla \psi_{j}^{\mathrm{KS} \dagger} \psi_{r}^{\mathrm{KS}}}{\psi_{j}^{\mathrm{KS} \dagger} \psi_{r}^{\mathrm{KS}}}-\frac{e}{m c} \mathbf{A}(\mathbf{r}, t), \\
\mathbf{m}^{(j, r)}(\mathbf{r}, t)=\psi_{j}^{\mathrm{KS} \dagger} \hat{\boldsymbol{\sigma}} \psi_{r}^{\mathrm{KS}} .
\end{gathered}
$$

Finally, from Eq. (A15) the divergence of the spin current tensor may be rewritten as

$$
-\nabla \cdot \mathbf{J}_{\mathrm{KS}}(\mathbf{r}, t)=\frac{\hbar}{2 \overline{\mathcal{F}} m} \nabla \cdot(n \mathbf{s} \times \nabla \mathbf{s})-\sum_{j \in \mathcal{O}} \sum_{l} \partial_{l}\left[\mathbf{m}_{j}(\mathbf{r}, t) \cdot v_{j}^{l}(\mathbf{r}, t)\right]-\frac{e}{m c} \sum_{l} \partial_{l}\left[n \mathbf{s} A^{l}(\mathbf{r}, t)\right]-\nabla \cdot \mathcal{D}(\mathbf{r}, t) .
$$

Then, by substituting Eq. (A20) into Eq. (A5) we obtain

$$
\frac{d}{d t} \mathbf{m}(\mathbf{r}, t)=-\nabla \cdot \mathcal{D}(\mathbf{r}, t)-\sum_{j \in \mathcal{O}} \sum_{l} \partial_{l}\left[\mathbf{m}_{j}(\mathbf{r}, t) \cdot v_{j}^{l}(\mathbf{r}, t)\right]+\frac{\hbar}{2 \overline{\mathcal{F}} m} \nabla \cdot(n \mathbf{s} \times \nabla \mathbf{s})+\mu_{B} n \mathbf{s} \times \mathbf{B}_{\mathrm{XC}}(\mathbf{r}, t)+\mathbf{T}_{\mathrm{SO}}(\mathbf{r}, t) .
$$

Finally, by decomposing the magnetization into its single-particle components $\mathbf{m}_{j}$ we can define the magnetization material derivative as

$$
\frac{D}{D t} \mathbf{m}(\mathbf{r}, t)=\frac{d}{d t} \sum_{j \in \mathcal{O}} \mathbf{m}_{j}(\mathbf{r}, t)+\sum_{j \in \mathcal{O}}\left(\mathbf{v}_{j} \cdot \nabla\right) \mathbf{m}_{j}(\mathbf{r}, t),
$$

with the spin continuity equation that becomes

$$
\frac{D}{D t} \mathbf{m}(\mathbf{r}, t)=-\nabla \cdot \mathcal{D}(\mathbf{r}, t)-\sum_{j \in \mathcal{O}} \nabla \cdot \mathbf{v}_{j}(\mathbf{r}, t) \mathbf{m}_{j}(\mathbf{r}, t)+\frac{\hbar}{2 \overline{\mathcal{F}} m} \nabla \cdot(n \mathbf{s} \times \nabla \mathbf{s})+\mu_{B} n \mathbf{s} \times \mathbf{B}_{\mathrm{XC}}(\mathbf{r}, t)+\mathbf{T}_{\mathrm{SO}}(\mathbf{r}, t)
$$

or

$$
\frac{D}{D t} \mathbf{m}(\mathbf{r}, t)+\sum_{j \in \mathcal{O}} \nabla \cdot \mathbf{v}_{j}(\mathbf{r}, t) \mathbf{m}_{j}(\mathbf{r}, t)=-\nabla \cdot \mathcal{D}(\mathbf{r}, t)+\mu_{B} \mathbf{m}(\mathbf{r}, t) \times \mathbf{B}_{\mathrm{eff}}(\mathbf{r}, t)+\mathbf{T}_{\mathrm{SO}}(\mathbf{r}, t),
$$

where we have introduced an effective magnetic field

$$
\mathbf{B}_{\text {eff }}[n, \mathbf{s}](\mathbf{r}, t)=\mathbf{B}_{\mathrm{XC}}[n, \mathbf{s}](\mathbf{r}, t)+\frac{1}{\overline{\mathcal{F}} e}\left[\frac{\nabla n \cdot \nabla \mathbf{s}}{n}+\nabla^{2} \mathbf{s}\right] .
$$

The continuity equation for the electron density instead follows immediately from Eq. (A4) through the definition of velocity field

$$
\frac{D n}{D t}=-n \nabla \cdot \mathbf{v}
$$

It should be noted that the kinetic field $\frac{1}{\mathcal{F} e}\left[\frac{\nabla n \cdot \nabla \mathbf{s}}{n}+\nabla^{2} \mathbf{s}\right]$ written in Eq. (A25) is expressed in terms of the density and spin density, which are observables of the many-body system. This means that the kinetic field obtained within the Kohn-Sham formalism is identical to its many-body counterpart.

[1] I. Tudosa, C. Stamm, A. B. Kashuba, F. King, H. C. Siegmann, J. Stohr, G. Ju, B. Lu, and D. Weller, Nature (London) 428, 831 (2004).

[2] T. Gerrits, H. A. M. Van den Berg, J. Hohlfeld, L. Bär, and T. Rasing, Nature (London) 418, 509 (2002).

[3] E. Beaurepaire, J.-C. Merle, A. Daunois, and J.-Y. Bigot, Phys. Rev. Lett. 76, 4250 (1996).

[4] A. V. Kimel, A. Kirilyuk, and T. Rasing, Laser Photon. Rev. 1, 275 (2007).

[5] A. Kirilyuk, A. V. Kimel, and T. Rasing, Rev. Mod. Phys. 82, 2731 (2010).
[6] B. Koopmans, G. Malinowski, F. Dalla Longa, D. Steiauf, M. Fähnle, T. Roth, M. Cinchetti, and M. Aeschlimann, Nat. Mater. 9, 259 (2010).

[7] Y. Hinschberger and P.-A. Hervieux, Phys. Lett. A 376, 813 (2012).

[8] H. Vonesch and J. Y. Bigot, Phys. Rev. B 85, 180407(R) (2012).

[9] B. Y. Mueller, A. Baral, S. Vollmar, M. Cinchetti, M. Aeschlimann, H. C. Schneider, and B. Rethfeld, Phys. Rev. Lett. 111, 167204 (2013).

[10] E. Carpene, E. Mancini, C. Dallera, M. Brenna, E. Puppin, and S. De Silvestri, Phys. Rev. B 78, 174422 (2008). 
[11] M. Krauss, T. Roth, S. Alebrand, D. Steil, M. Cinchetti, M. Aeschlimann, and H. C. Schneider, Phys. Rev. B 80, 180407(R) (2009).

[12] M. Battiato, K. Carva, and P. M. Oppeneer, Phys. Rev. B 86, 024404 (2012).

[13] E. Runge and E. K. U. Gross, Phys. Rev. Lett. 52, 997 (1984).

[14] E. K. U. Gross and W. Kohn, Adv. Quantum Chem. 21, 255 (1990).

[15] K. Krieger, J. K. Dewhurst, P. Elliott, S. Sharma, and E. K. U. Gross, J. Chem. Theory Comput. 11, 4870 (2015).

[16] W. Töws and G. M. Pastor, Phys. Rev. Lett. 115, 217204 (2015).

[17] G. P. Zhang, Phys. Rev. Lett. 101, 187203 (2008).

[18] M. Stamenova, J. Simoni, and S. Sanvito, Phys. Rev. B 94, 014423 (2016).

[19] V. Antropov, J. Appl. Phys. 97, 10A704 (2005).

[20] M. I. Katsnelson and V. P. Antropov, Phys. Rev. B 67, 140406(R) (2003).

[21] L. Fernandez-Seivane, M. A. Oliveira, S. Sanvito, and J. Ferrer, J. Phys.: Condens. Matter 18, 7999 (2006).

[22] M. Levy and J. P. Perdew, Phys. Rev. A 32, 2010 (1985).

[23] K. Capelle, G. Vignale, and B. L. Györffy, Phys. Rev. Lett. 87, 206403 (2001).

[24] S. Sharma, J. K. Dewhurst, C. Ambrosch-Draxl, S. Kurth, N. Helbig, S. Pittalis, S. Shallcross, L. Nordström, and E. K. U. Gross, Phys. Rev. Lett. 98, 196405 (2007).

[25] F. G. Eich and E. K. U. Gross, Phys. Rev. Lett. 111, 156401 (2013).
[26] S. Sharma, S. Pittalis, S. Kurth, S. Shallcross, J. K. Dewhurst, and E. K. U. Gross, Phys. Rev. B 76, 100401 (2007).

[27] T. Koide, Phys. Rev. C 87, 034902 (2013).

[28] M. Marklund and G. Brodin, Phys. Rev. Lett. 98, 025001 (2007).

[29] T. Takabayasi, Prog. Theor. Phys. 14, 283 (1955).

[30] T. Takabayasi and J.-P. Vigier, Prog. Theor. Phys. 18, 573 (1957).

[31] A. Castro, H. Appel, M. Oliveira, C. A. Rozzi, X. Andrade, F. Lorenzen, M. A. L. Marques, E. K. U. Gross, and A. Rubio, Phys. Status Solidi B 243, 2465 (2006).

[32] C. L. Reis, J. M. Pacheco, and J. L. Martins, Phys. Rev. B 68, 155111 (2003).

[33] M. J. T. Oliveira and F. Nogueira, Comput. Phys. Commun. 178, 524 (2008).

[34] M. A. L. Marques, M. J. T. Oliveira, and T. Burnus, Comput. Phys. Commun. 183, 2272 (2012).

[35] J. Zhu, X. W. Wang, and S. G. Louie, Phys. Rev. B 45, 8887 (1992).

[36] O. Diéguez, M. M. G. Alemany, C. Rey, P. Ordejón, and L. J. Gallego, Phys. Rev. B 63, 205407 (2001).

[37] G. L. Gutsev and C. W. Bauschlicher, Jr., J. Chem. Phys. 107, 7013 (2003).

[38] P. R. Holland, The Quantum Theory of Motion (Cambridge University Press, Cambridge, 1993).

[39] M. Schönberg, Nuovo Cimento 12, 103 (1954).

[40] G. Brodin, M. Marklund, J. Zamanian, and M. Stefan, Plasma Phys. Contr. Fusion 53, 074013 (2011). 